\title{
Hydrological response characteristics and classification of Lebanese catchments
}

\author{
Mohammed Merheb $^{1 *}$, Chadi Abdallah ${ }^{2}$, Roger Moussa $^{3}$, Nicolas Baghdadi $^{4}$ \\ ${ }^{(1)}$ Lebanese University, Faculty of Sciences 3, Tripoli, Lebanon. \\ ${ }^{(2)}$ CNRS-L, Beirut, Lebanon. \\ ${ }^{(3)}$ LISAH, Univ. Montpellier, INRA, IRD, SupAgro, Montpellier, France. \\ (4) Inrae, Montpellier, France \\ * Corresponding author at: Mohammad Merheb, Lebanese University, Tripoli, Lebanon \\ E-mail address: mohammadmerheb1987@gmail.com
}

The Mediterranean region is very vulnerable to both water shortage and flooding. Hence, the study of the hydrological response characteristics of Mediterranean catchments is of a great importance especially that the region is one of the most densely populated on the planet. Numerous Mediterranean catchment hydrological studies exist in the literature, however, mostly concentrated in the European part of the Mediterranean, especially the north-western part of the basin. The eastern Mediterranean have fewer studies and this is indeed the case of Lebanon, a small mountainous country on the eastern shore of the Mediterranean. The objective of this work is to present a state of the art assessment of the hydrology of Lebanon in the largest Mediterranean context and to present a first classification attempt for the Lebanese catchments. Twenty-eight Lebanese basins where data are available are studied. Spatial data consist of Digital Elevation Model, land cover map, soil and geological maps of the country. Temporal hydrometeorological data exist at a monthly and daily timescale for the period 2001-2011. The methodology is threefold: (1) a synthesis of the previous hydrological studies in Lebanon, (2) an analysis of the hydrological response characteristics of the Lebanese catchments and a comparison with other Mediterranean catchments, and (3) the classification of the Lebanese catchments according to their physical and hydrological response characteristics. After extracting physical descriptors and runoff signatures for the available data, using Agglomerative Hierarchical Clustering. The results of the synthesis of previous studies in Lebanon assert that indeed hydrological studies in Lebanon are scarce and when exist focus mostly on groundwater hydrology and water quality. Nevertheless. The Lebanese literature suggest that even though rainfall and physical characteristics are highly variable across the country, a certain regional pattern do exist. This is corroborated by the analysis of the Lebanese catchments temporal data. Indeed, mean annual runoff and runoff ratio across the country show regional 
tendencies with the highest values in the central part of mount Lebanon. Moreover, intra-annual variation of runoff also shows geographical pattern and can be divided into three main regimes: rainfall dominated, snow dominated and a mixed snow-rainfall regime. This is also true at the event scale, where unit maximum daily discharge and runoff depth and ratio, all show regional pattern with the highest values in the central part of Lebanon which receive the highest precipitation amount. In addition, the Lebanese catchments, and despite a high reference evapotranspiration, exhibit hydrological response characteristics that are similar to catchments in the wetter north-western Mediterranean than other catchments in the Eastern Mediterranean. Finally, the classification of the Lebanese catchments yielded five groups of catchments. This grouping is well in range with the regional pattern discussed in the literature and the data analysis of the Lebanese catchments. 